Partially polarized Gaussian Schell-model beams

This article has been downloaded from IOPscience. Please scroll down to see the full text article.

2001 J. Opt. A: Pure Appl. Opt. 31

(http://iopscience.iop.org/1464-4258/3/1/301)

View the table of contents for this issue, or go to the journal homepage for more

Download details:

IP Address: 147.96.14.16

The article was downloaded on 11/06/2013 at 18:33

Please note that terms and conditions apply. 


\title{
Partially polarized Gaussian Schell-model beams
}

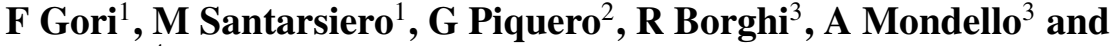 \\ R Simon ${ }^{4}$
}

\author{
${ }^{1}$ Dipartimento di Fisica, Istituto Nazionale per la Fisica della Materia, Università 'Roma \\ Tre', Via della Vasca Navale 84, I-00146 Rome, Italy \\ 2 Departamento de Óptica, Universidad Complutense de Madrid, Ciudad Universitaria s/n, \\ E-28040 Madrid, Spain \\ ${ }^{3}$ Dipartimento di Ingegneria Elettronica, Istituto Nazionale per la Fisica della Materia, \\ Università 'Roma Tre', Via della Vasca Navale 84, I-00146 Rome, Italy \\ ${ }^{4}$ The Institute of Mathematical Sciences, CIT Campus, Tharamani, Chennai 600 113, India
}

Received 9 May 2000, in final form 3 October 2000

\begin{abstract}
We consider a class of beams that are both partially polarized and partially coherent from the spatial standpoint. They are characterized by a correlation matrix whose elements have the same form as the mutual intensity of a Gaussian Schell-model beam. We focus our attention on those beams that would appear identical to ordinary Gaussian Schell-model beams in a scalar treatment. After establishing some inequalities that limit the choice of the matrix parameters, we study the main effects of propagation. Starting from the source plane, in which the beam is assumed to be uniformly polarized, we find that in the course of propagation the degree of polarization generally becomes non-uniform across a typical section of the beam. Furthermore, we find that the intensity distribution at the output of an arbitrarily oriented linear polarizer is Gaussian shaped at the source plane whereas it can be quite different at other planes.
\end{abstract}

Keywords: Polarization, beams, coherence theory

\section{Introduction}

Partially coherent beams characterized by a mutual intensity (or by a cross-spectral density) of the Gaussian Schell-model (GSM) type [1] have played a very important role in coherence theory $[1,2]$. To quote just a few examples, they were widely used for investigating the relationship between radiometry and coherence theory [3-18], offered the first explicit example [1922] of modal expansion of the cross-spectral density $[1,23]$ and were crucial to introducing the concept of twisting for partially coherent beams [24-30]. The interest in these beams is far from subsiding as proved by recent papers [31,32]. The previously quoted investigations used a scalar description. The underlying assumption is that the state of polarization of the beam does not play any significant role in the phenomena under scrutiny. A typical case in which this can be assumed is when the beam is completely and uniformly polarized. We shall refer to the beam considered in the scalar treatment as the ordinary GSM beam. On the other hand, light beams with non-uniform and/or partial polarization have gained considerable attention in recent times [33-53]. Therefore, it seems of interest to inquire about the possibility of using a suitable extension of the GSM structure when passing from a scalar to a vectorial treatment. In other words, it is worthwhile to investigate beams that are both partially polarized and partially coherent from the spatial standpoint when the correlation functions among different field components have a GSM form. For the sake of brevity, we shall refer to such beams as partially polarized GSM (or PGSM) beams.

In this paper, we discuss certain basic properties of PGSM beams, making use of a description based on the BCP (beam coherence-polarization) matrix $[54,55]$. As will be seen, the matrix elements are specified by nine parameters and this makes the general discussion rather demanding. On the other hand, we can focus our attention on a particular class of beams. Indeed, it is physically interesting to answer the following question: is there any partially polarized beam that behaves like an ordinary GSM beam if polarization is ignored and yet is physically different from the ordinary GSM beam? In order to clarify the meaning of this question we add that a PGSM beam is said to behave as an ordinary GSM beam in the scalar sense if its equivalent mutual intensity [55,56] (in particular 
its optical intensity) is identical to that of an ordinary GSM beam at any transverse plane in the course of propagation. In addition, for the sake of simplicity, we shall require the degree of polarization to be uniform across the source plane.

At first, we could think that having imposed such constraints the resulting PGSM beam should not be very different from an ordinary one. In contrast, we shall see that significant differences can be found when polarization properties are taken into account. For example, the degree of polarization, although uniform across the source plane, becomes a function of position upon propagation. Even more intriguing and easier to demonstrate is the effect of a linear polarizer inserted in the beam path. If we place the polarizer against the source the emerging intensity distribution is Gaussian shaped irrespective of how the polarizer is oriented. On the other hand, if the polarizer is inserted at a different plane the output intensity distribution changes upon rotation of the polarizer and is generally non-Gaussian. Strange as it may sound, all this is a consequence of the GSM form for the elements of the BCP matrix.

Something has to be added about the choice of the parameters characterizing the matrix elements. On limiting ourselves to partially polarized beams that are undistinguishable from ordinary GSM beams when polarization is disregarded, and using the hypothesis that the degree of polarization is uniform across the source plane, we reduce the number of free parameters from nine to six. Furthermore, these six parameters cannot be chosen at will, since certain inequalities have to be satisfied. Surprisingly enough, it turns out that, except for particular cases, the correlation between the $x$ - and $y$-components of the electric field must have a greater range (correlation length) than the self-correlation of the $x$ - (or $y$-) component.

The paper is organized as follows. After a few preliminaries devoted to recollecting the main formulae relating to the $\mathrm{BCP}$ treatment (section 2), we shall characterize the class of PGSM beams of our interest (section 3.1). Then, we shall discuss the inequalities that the parameters of the matrix elements must meet (section 3.2). The most relevant effects of the propagation process on the polarization characteristics are presented in section 4 with the aid of a numerical example (section 5). Some final remarks and hints for extensions are given in section 6 .

\section{Preliminaries}

We shall use a reference frame in which the $z$-axis coincides with the mean direction of propagation of the beam. At a typical transverse plane, the vector $r$ is used to specify the position of a point. Let us recall that, for a quasimonochromatic field, the BCP matrix is defined as [54, 55]

$$
\hat{J}\left(\boldsymbol{r}_{1}, \boldsymbol{r}_{2}, z\right)=\left(\begin{array}{cc}
J_{x x}\left(\boldsymbol{r}_{1}, \boldsymbol{r}_{2}, z\right) & J_{x y}\left(\boldsymbol{r}_{1}, \boldsymbol{r}_{2}, z\right) \\
J_{y x}\left(\boldsymbol{r}_{1}, \boldsymbol{r}_{2}, z\right) & J_{y y}\left(\boldsymbol{r}_{1}, \boldsymbol{r}_{2}, z\right)
\end{array}\right),
$$

where

$$
\begin{gathered}
J_{\alpha \beta}\left(\boldsymbol{r}_{1}, \boldsymbol{r}_{2}, z\right)=\left\langle E_{\alpha}^{*}\left(\boldsymbol{r}_{1}, z ; t\right) E_{\beta}\left(\boldsymbol{r}_{2}, z ; t\right)\right\rangle, \\
(\alpha, \beta=x, y) .
\end{gathered}
$$

The angle brackets denote time average and $E_{\alpha}(\alpha=x, y)$ is a Cartesian component of the time-dependent electric field. The
$\mathrm{BCP}$ matrix gives an approximate form of the general tensorial theory of coherence developed by Wolf $[1,57]$.

The following relationships for the $\mathrm{BCP}$ matrix hold:

$$
\begin{gathered}
J_{x y}\left(\boldsymbol{r}_{1}, \boldsymbol{r}_{2}, z\right)=J_{y x}^{*}\left(\boldsymbol{r}_{2}, \boldsymbol{r}_{1}, z\right), \\
\left|J_{\alpha \beta}\left(\boldsymbol{r}_{1}, \boldsymbol{r}_{2}, z\right)\right|^{2} \leqslant J_{\alpha \alpha}\left(\boldsymbol{r}_{1}, \boldsymbol{r}_{1}, z\right) J_{\beta \beta}\left(\boldsymbol{r}_{2}, \boldsymbol{r}_{2}, z\right), \\
(\alpha, \beta=x, y) .
\end{gathered}
$$

Furthermore, the following important non-negativity condition has to be satisfied:

$$
\begin{aligned}
\iint & {\left[f_{1}^{*}\left(\boldsymbol{r}_{1}\right) f_{1}\left(\boldsymbol{r}_{2}\right) J_{x x}\left(\boldsymbol{r}_{1}, \boldsymbol{r}_{2}, z\right)\right.} \\
& +f_{1}^{*}\left(\boldsymbol{r}_{1}\right) f_{2}\left(\boldsymbol{r}_{2}\right) J_{x y}\left(\boldsymbol{r}_{1}, \boldsymbol{r}_{2}, z\right) \\
& +f_{2}^{*}\left(\boldsymbol{r}_{1}\right) f_{1}\left(\boldsymbol{r}_{2}\right) J_{y x}\left(\boldsymbol{r}_{1}, \boldsymbol{r}_{2}, z\right) \\
& \left.+f_{2}^{*}\left(\boldsymbol{r}_{1}\right) f_{2}\left(\boldsymbol{r}_{2}\right) J_{y y}\left(\boldsymbol{r}_{1}, \boldsymbol{r}_{2}, z\right)\right] \mathrm{d}^{2} r_{1} \mathrm{~d}^{2} r_{2} \geqslant 0,
\end{aligned}
$$

where $f_{1}(\boldsymbol{r})$ and $f_{2}(\boldsymbol{r})$ are arbitrary functions.

The local polarization properties of the beam at a typical point of a cross section are specified by the BCP matrix with $\boldsymbol{r}_{1}=\boldsymbol{r}_{2}=\boldsymbol{r}$. We shall refer to it as the local polarization matrix. Further, the degree of polarization $[1,58,59]$ is given by [55]

$$
P(r, z)=\sqrt{1-\frac{4 \operatorname{det}\{\hat{J}(r, r, z)\}}{(\operatorname{Tr}\{\hat{J}(\boldsymbol{r}, \boldsymbol{r}, z)\})^{2}}},
$$

where det stands for the determinant and Tr denotes the trace of the BCP matrix.

In addition, we can define an equivalent mutual intensity as

$$
\begin{aligned}
J_{\text {eq }}\left(\boldsymbol{r}_{1}, \boldsymbol{r}_{2}, z\right) & =J_{x x}\left(\boldsymbol{r}_{1}, \boldsymbol{r}_{2}, z\right)+J_{y y}\left(\boldsymbol{r}_{1}, \boldsymbol{r}_{2}, z\right) \\
& =\operatorname{Tr}\left\{\hat{J}\left(\boldsymbol{r}_{1}, \boldsymbol{r}_{2}, z\right)\right\} .
\end{aligned}
$$

In fact, if no anisotropic element is used, all propagation, diffraction and interference phenomena will be correctly described by replacing the actual beam by one describable in scalar terms, provided that the mutual intensity of the latter is given by equation $(7)[55,56]$.

In particular, the optical intensity at a typical point, say $I(r, z)$, will be given by

$$
I(\boldsymbol{r}, z)=J_{\mathrm{eq}}(\boldsymbol{r}, \boldsymbol{r}, z) .
$$

If some anisotropic optical element, whose Jones matrix $[1,58,59]$ is denoted by $\hat{T}(\boldsymbol{r})$, is inserted in the beam path at a certain plane $z=$ constant, then the BCP matrix of the field emerging from it, say $\hat{J}^{\prime}$, can be evaluated [55] through the relation

$$
\hat{J}^{\prime}\left(\boldsymbol{r}_{1}, \boldsymbol{r}_{2}, z\right)=\hat{T}^{\dagger}\left(\boldsymbol{r}_{1}\right) \hat{J}\left(\boldsymbol{r}_{1}, \boldsymbol{r}_{2}, z\right) \hat{T}\left(\boldsymbol{r}_{2}\right),
$$

where the dagger denotes the Hermitian conjugate. For instance, the intensity emerging from a uniform linear polarizer, whose transmission axis forms an angle $\vartheta$ (anticlockwise) with the $x$-axis, turns out to be

$$
\begin{aligned}
& I_{\vartheta}(\boldsymbol{r}, z)=J_{x x}(\boldsymbol{r}, \boldsymbol{r}, z) C^{2} \\
& \quad+J_{y y}(\boldsymbol{r}, \boldsymbol{r}, z) S^{2}+2 \operatorname{Re}\left\{J_{x y}(\boldsymbol{r}, \boldsymbol{r}, z)\right\} C S
\end{aligned}
$$

where $C=\cos \vartheta$ and $S=\sin \vartheta$, and Re stands for the real-part operator.

Our present treatment applies to quasimonochromatic fields. Its extension to the polychromatic case involves the use of the cross-spectral density [1]. Most of the results we are going to derive could be easily translated into the language of the space-frequency description. 


\section{Partially polarized GSM beams}

\subsection{Definitions and notations}

We recall that the mutual intensity of an ordinary, scalar GSM source, placed at $z=0$, say $J_{\mathrm{sc}}\left(\boldsymbol{r}_{1}, \boldsymbol{r}_{2}, 0\right)$, takes the form

$$
J_{\mathrm{sc}}\left(\boldsymbol{r}_{1}, \boldsymbol{r}_{2}, 0\right)=I_{0} \exp \left[-\frac{r_{1}^{2}+r_{2}^{2}}{4 \sigma_{I_{0}}^{2}}-\frac{\left(\boldsymbol{r}_{1}-\boldsymbol{r}_{2}\right)^{2}}{2 \sigma_{\mu_{0}}^{2}}\right],
$$

where $I_{0}$ is a constant intensity factor, while $\sigma_{I_{0}}^{2}$ and $\sigma_{\mu_{0}}^{2}$ are the widths of the transverse intensity profile and of the degree of coherence, respectively.

It seems natural to define a PGSM source as one in which all the elements of the BCP matrix have a form similar to equation (11). More explicitly, we let

$$
\begin{gathered}
J_{\alpha \beta}\left(\boldsymbol{r}_{1}, \boldsymbol{r}_{2}, 0\right)=I_{0 \alpha \beta} \exp \left[-\frac{r_{1}^{2}+r_{2}^{2}}{4 \sigma_{I_{0 \alpha \beta}}^{2}}-\frac{\left(\boldsymbol{r}_{1}-\boldsymbol{r}_{2}\right)^{2}}{2 \sigma_{\mu_{0 \alpha \beta}}^{2}}\right], \\
(\alpha, \beta=x, y) .
\end{gathered}
$$

It should be noted that $I_{0 x x}$ and $I_{0 y y}$ have to be real, positive quantities, while $I_{0 x y}$ may be complex. Due to equation (3), $I_{0 y x}=I_{0 x y}^{*}$. In physical terms, the argument of $I_{0 x y}$ accounts for a constant phase shift between the $x$ - and $y$-components of the field. Such a phase difference could be easily eliminated using a suitable waveplate (see appendix A). Accordingly, for the sake of simplicity, the argument of $I_{0 x y}$ will be set to zero. It also follows from equation (3) that

$$
\sigma_{I_{0 x y}}=\sigma_{I_{0 y x}} ; \quad \sigma_{\mu_{0 x y}}=\sigma_{\mu_{0 y x}} .
$$

It is seen that the matrix elements (12) are specified by a set of nine real parameters. Beams generated by this type of source have already been considered by some authors [37,53].

As we said in the introduction, we shall now impose that, in scalar terms, the PGSM source must be indistinguishable from an ordinary, scalar GSM source. This means that the equivalent mutual intensity $J_{\text {eq }}\left(\boldsymbol{r}_{1}, \boldsymbol{r}_{2}, 0\right)$ associated with the BCP matrix (see equation (7)) must be equal to $J_{\mathrm{sc}}\left(\boldsymbol{r}_{1}, \boldsymbol{r}_{2}, 0\right)$, for any choice of $\boldsymbol{r}_{1}, \boldsymbol{r}_{2}$. On using equations (7), (11) and (12) we find at once the following conditions:

$$
\sigma_{I_{0 x x}}=\sigma_{I_{0 y y}} ; \quad \sigma_{\mu_{0 x x}}=\sigma_{\mu_{0 y y}},
$$

while $I_{0 x x}$ and $I_{0 y y}$ can be different.

Using equations (6) and (12) we easily derive, for the local degree of polarization at the source plane, the following expression:

$$
\begin{aligned}
& P(\boldsymbol{r}, 0) \\
& =\left\{\left(\frac{I_{0 x x}-I_{0 y y}}{I_{0 x x}+I_{0 y y}}\right)^{2}\right. \\
& \left.\quad+\frac{4 I_{0 x y}^{2}}{\left(I_{0 x x}+I_{0 y y}\right)^{2}} \exp \left[-\left(\frac{1}{\sigma_{I_{0 x y}^{2}}^{2}}-\frac{1}{\sigma_{I_{0 x x}}^{2}}\right) r^{2}\right]\right\}^{1 / 2} .
\end{aligned}
$$

Furthermore, we require the degree of polarization to be uniform across the source plane, i.e. independent of $r$. By virtue of equation (15) this implies

$$
\sigma_{I_{0 x y}}=\sigma_{I_{0 x x}} .
$$

Taking equation (14) into account, we see that $\sigma_{I_{0 x x}}, \sigma_{I_{0 y y}}$ and $\sigma_{I_{0 x y}}$ have the same value. We shall use for this common value the same symbol, $\sigma_{I_{0}}$, pertaining to the scalar source. Using equations (15) and (16) we see that the local degree of polarization across the source plane is now expressed by

$$
P(\boldsymbol{r}, 0)=\sqrt{\left(\frac{I_{0 x x}-I_{0 y y}}{I_{0 x x}+I_{0 y y}}\right)^{2}+\frac{4 I_{0 x y}^{2}}{\left(I_{0 x x}+I_{0 y y}\right)^{2}}} .
$$

Taking into account the inequality

$$
I_{0 x y}^{2} \leqslant I_{0 x x} I_{0 y y}
$$

which follows from equations (4) and (12), we can easily control that $P \leqslant 1$. The number of free parameters is now reduced to six. They are $I_{0 x x}, I_{0 y y}, I_{0 x y}, \sigma_{I_{0}}, \sigma_{\mu_{0 x x}}$ and $\sigma_{\mu_{0 x y}}$. However, such parameters cannot be chosen completely at will. One constraint is expressed by equation (18). Others will be seen in a moment.

\subsection{Non-negativity of the BCP matrix}

We now examine the constraints connected to the nonnegativity condition (5). In particular, we derive a necessary condition and a sufficient condition for the choice of the free parameters.

First of all, we let $f_{1}(\boldsymbol{r})=-f_{2}(\boldsymbol{r})=f(\boldsymbol{r})$ in equation (5), with

$$
f(\boldsymbol{r})=\exp (\mathrm{i} 2 \pi \boldsymbol{u} \cdot \boldsymbol{r}),
$$

where $\boldsymbol{u}$ denotes a typical vector in the spatial frequency plane and the dot represents the standard inner product. This choice will lead us to a necessary condition.

Let us write the BCP elements in the form

$$
\begin{gathered}
J_{\alpha \beta}\left(\boldsymbol{r}_{1}, \boldsymbol{r}_{2} ; 0\right)=I_{0 \alpha \beta} \exp \left[-p\left(\boldsymbol{r}_{1}+\boldsymbol{r}_{2}\right)^{2}-m_{\alpha \beta}\left(\boldsymbol{r}_{1}-\boldsymbol{r}_{2}\right)^{2}\right], \\
(\alpha, \beta=x, y),
\end{gathered}
$$

where

$$
p=\frac{1}{8 \sigma_{I_{0}}^{2}} ; \quad m_{\alpha \beta}=\frac{1}{8 \sigma_{I_{0}}^{2}}+\frac{1}{2 \sigma_{\mu_{0 \alpha \beta}}^{2}} .
$$

Then, equation (5) becomes

$$
\begin{aligned}
& \iint \exp \left[-\mathrm{i} 2 \pi \boldsymbol{u} \cdot\left(\boldsymbol{r}_{1}-\boldsymbol{r}_{2}\right)-p\left(\boldsymbol{r}_{1}+\boldsymbol{r}_{2}\right)^{2}\right] \\
& \quad \times\left\{\left(I_{0 x x}+I_{0 y y}\right) \exp \left[-m_{x x}\left(\boldsymbol{r}_{1}-\boldsymbol{r}_{2}\right)^{2}\right]\right. \\
& \left.\quad-2 I_{0 x y} \exp \left[-m_{x y}\left(\boldsymbol{r}_{1}-\boldsymbol{r}_{2}\right)^{2}\right]\right\} \mathrm{d}^{2} r_{1} \mathrm{~d}^{2} r_{2} \geqslant 0 .
\end{aligned}
$$

The integral in equation (22) can be evaluated on introducing the variables

$$
s=\frac{r_{1}+r_{2}}{2} ; \quad t=r_{1}-r_{2},
$$

giving

$$
\begin{array}{r}
\frac{\pi^{2}}{4 p}\left\{\frac{\left(I_{0 x x}+I_{0 y y}\right)}{m_{x x}} \exp \left(-\frac{\pi^{2} u^{2}}{m_{x x}}\right)\right. \\
\left.-\frac{2 I_{0 x y}}{m_{x y}} \exp \left(-\frac{\pi^{2} u^{2}}{m_{x y}}\right)\right\} \geqslant 0,
\end{array}
$$


for any choice of $u$. In particular, for $u=0$ and for $u \rightarrow \infty$ condition (24) leads to

$$
\frac{I_{0 x x}+I_{0 y y}}{m_{x x}} \geqslant \frac{2 I_{0 x y}}{m_{x y}},
$$

and

$$
m_{x x} \geqslant m_{x y}
$$

respectively. Furthermore, on taking equation (21) into account, equations (25) and (26) become

$$
\frac{I_{0 x x}+I_{0 y y}}{2 I_{0 x y}} \geqslant \frac{\sigma_{\mu_{0 x y}}^{2}}{\sigma_{\mu_{0 x x}}^{2}} \frac{4 \sigma_{I_{0}}^{2}+\sigma_{\mu_{0 x x}}^{2}}{4 \sigma_{I_{0}}^{2}+\sigma_{\mu_{0 x y}}^{2}},
$$

and

$$
\sigma_{\mu_{0 x x}} \leqslant \sigma_{\mu_{0 x y}},
$$

respectively. Finally, from equations (14) and (16) we have

$$
\sigma_{\mu_{0 x x}} \leqslant \sigma_{\mu_{0 x y}} \leqslant \sigma_{\mu_{0 x x}} \sqrt{\frac{I_{0 x x}+I_{0 y y}}{2 I_{0 x y}} \frac{4 \sigma_{I_{0}}^{2}+\sigma_{\mu_{0 x y}}^{2}}{4 \sigma_{I_{0}}^{2}+\sigma_{\mu_{0 x x}}^{2}}},
$$

which represents a necessary condition for the parameters of the PGSM source. The upper bound has an implicit form. We do not dwell on transforming it into an explicit form, because the most important result is given by the lower bound. The latter, in particular, implies that the correlation length pertaining to the off-diagonal terms of the BCP matrix cannot be lower than that pertaining to the diagonal terms. This may sound surprising. However, it was already noted that $J_{x y}\left(\boldsymbol{r}_{1}, \boldsymbol{r}_{2}, z\right)$ is not locally connected to $J_{x x}\left(\boldsymbol{r}_{1}, \boldsymbol{r}_{2}, z\right)$ except when $\boldsymbol{r}_{1}=\boldsymbol{r}_{2}$ [55].

Let us now derive a sufficient condition. Starting again from equation (5), and letting

$$
g_{j}(\boldsymbol{r})=f_{j}(\boldsymbol{r}) \exp \left(-\frac{r^{2}}{4 \sigma_{I o}^{2}}\right), \quad(j=1,2),
$$

equation (5) turns out to be

$$
\begin{aligned}
\iint & \left\{\left[I_{0 x x} g_{1}^{*}\left(\boldsymbol{r}_{1}\right) g_{1}\left(\boldsymbol{r}_{2}\right)+I_{0 y y} g_{2}^{*}\left(\boldsymbol{r}_{1}\right) g_{2}\left(\boldsymbol{r}_{2}\right)\right]\right. \\
& \times \exp \left[-\frac{\left(\boldsymbol{r}_{1}-\boldsymbol{r}_{2}\right)^{2}}{2 \sigma_{\mu_{0 x x}}^{2}}\right] \\
& +I_{0 x y}\left[g_{1}^{*}\left(\boldsymbol{r}_{1}\right) g_{2}\left(\boldsymbol{r}_{2}\right)+g_{2}^{*}\left(\boldsymbol{r}_{1}\right) g_{1}\left(\boldsymbol{r}_{2}\right)\right] \\
& \left.\times \exp \left[-\frac{\left(\boldsymbol{r}_{1}-\boldsymbol{r}_{2}\right)^{2}}{2 \sigma_{\mu_{0 x y}}^{2}}\right]\right\} \mathrm{d}^{2} r_{1} \mathrm{~d}^{2} r_{2} \geqslant 0 .
\end{aligned}
$$

On expressing the Gaussian functions depending on $\boldsymbol{r}_{1}-\boldsymbol{r}_{2}$ by means of their Fourier transform, equation (31) becomes

$$
\begin{aligned}
& \iiint\left\{\left[I_{0 x x} g_{1}^{*}\left(\boldsymbol{r}_{1}\right) g_{1}\left(\boldsymbol{r}_{2}\right)\right.\right. \\
& \left.\quad+I_{0 y y} g_{2}^{*}\left(\boldsymbol{r}_{1}\right) g_{2}\left(\boldsymbol{r}_{2}\right)\right] \sigma_{\mu_{0 x x}}^{2} \exp \left(-2 \pi^{2} \sigma_{\mu_{0 x x}}^{2} u^{2}\right) \\
& \quad+I_{0 x y}\left[g_{1}^{*}\left(\boldsymbol{r}_{1}\right) g_{2}\left(\boldsymbol{r}_{2}\right)+g_{2}^{*}\left(\boldsymbol{r}_{1}\right) g_{1}\left(\boldsymbol{r}_{2}\right)\right] \\
& \left.\quad \times \sigma_{\mu_{0 x y}}^{2} \exp \left(-2 \pi^{2} \sigma_{\mu_{0 x y}}^{2} u^{2}\right)\right\} \\
& \quad \times \exp \left[\mathrm{i} 2 \pi\left(\boldsymbol{r}_{1}-\boldsymbol{r}_{2}\right) \cdot \boldsymbol{u}\right] \mathrm{d}^{2} r_{1} \mathrm{~d}^{2} r_{2} \mathrm{~d}^{2} u \geqslant 0 .
\end{aligned}
$$

If we now introduce the Fourier transform of the functions $g_{j}(\boldsymbol{r})$, namely $\tilde{g}_{j}(\boldsymbol{u})(j=1,2)$, we obtain

$$
\begin{gathered}
\int\left\{\left[I_{0 x x}\left|\tilde{g}_{1}(\boldsymbol{u})\right|^{2}+I_{0 y y}\left|\tilde{g}_{2}(\boldsymbol{u})\right|^{2}\right] \exp \left(-2 \pi^{2} \sigma_{\mu_{0 x x}}^{2} u^{2}\right)\right. \\
+2\left(\frac{\sigma_{\mu_{0 x y}}}{\sigma_{\mu_{0 x x}}}\right)^{2} I_{0 x y} \operatorname{Re}\left[\tilde{g}_{1}^{*}(\boldsymbol{u}) \tilde{g}_{2}(\boldsymbol{u})\right] \\
\left.\quad \times \exp \left(-2 \pi^{2} \sigma_{\mu_{0 x y}}^{2} u^{2}\right)\right\} \mathrm{d}^{2} u \geqslant 0,
\end{gathered}
$$

which can be written as

$$
\begin{aligned}
\int\{ & {\left[\left|\sqrt{I_{0 x x}} \tilde{g}_{1}(\boldsymbol{u})\right|^{2}+\left|\sqrt{I_{0 y y}} \tilde{g}_{2}(\boldsymbol{u})\right|^{2}\right] } \\
& \times \exp \left(-2 \pi^{2} \sigma_{\mu_{0 x x}^{2}}^{2} u^{2}\right)+2\left(\frac{\sigma_{\mu_{0 x y}}}{\sigma_{\mu_{0 x x}}}\right)^{2} \frac{I_{0 x y}}{\sqrt{I_{0 x x} I_{0 y y}}} \\
& \times \operatorname{Re}\left[\sqrt{I_{0 x x}} \tilde{g}_{1}^{*}(\boldsymbol{u}) \sqrt{I_{0 y y}} \tilde{g}_{2}(\boldsymbol{u})\right] \\
& \left.\times \exp \left(-2 \pi^{2} \sigma_{\mu_{0 x y}}^{2} u^{2}\right)\right\} \mathrm{d}^{2} u \geqslant 0 .
\end{aligned}
$$

Furthermore, taking the following identity into account:

$\left(|a|^{2}+|b|^{2}\right) U_{1}+2 \operatorname{Re}\left\{a^{*} b\right\} U_{2}$

$$
=\frac{1}{2}|a+b|^{2}\left(U_{1}+U_{2}\right)+\frac{1}{2}|a-b|^{2}\left(U_{1}-U_{2}\right),
$$

where $a$ and $b$ are arbitrary complex numbers, while $U_{1}$ and $U_{2}$ are real positive numbers, equation (33) can be written

$$
\begin{aligned}
\int \mid & \sqrt{I_{0 x x}} \tilde{g}_{1}(\boldsymbol{u})+\left.\sqrt{I_{0 y y}} \tilde{g}_{2}(\boldsymbol{u})\right|^{2} \\
& \times\left[\exp \left(-2 \pi^{2} \sigma_{\mu_{0 x x}}^{2} u^{2}\right)+\left(\frac{\sigma_{\mu_{0 x y}}}{\sigma_{\mu_{0 x x}}}\right)^{2} \frac{I_{0 x y}}{\sqrt{I_{0 x x} I_{0 y y}}}\right. \\
& \left.\times \exp \left(-2 \pi^{2} \sigma_{\mu_{0 x y}}^{2} u^{2}\right)\right] \mathrm{d}^{2} u \\
& +\int\left|\sqrt{I_{0 x x}} \tilde{g}_{1}(\boldsymbol{u})-\sqrt{I_{0 y y}} \tilde{g}_{2}(\boldsymbol{u})\right|^{2} \\
& \times\left[\exp \left(-2 \pi^{2} \sigma_{\mu_{0 x x}}^{2} u^{2}\right)-\left(\frac{\sigma_{\mu_{0 x y}}}{\sigma_{\mu_{0 x x}}}\right)^{2} \frac{I_{0 x y}}{\sqrt{I_{0 x x} I_{0 y y}}}\right. \\
& \left.\times \exp \left(-2 \pi^{2} \sigma_{\mu_{0 x y}}^{2} u^{2}\right)\right] \mathrm{d}^{2} u \geqslant 0 .
\end{aligned}
$$

The first integral in equation (36) is always positive. Therefore, a sufficient condition for satisfying inequality (36) for any choice of $f_{1}$ and $f_{2}$ is

$$
\begin{aligned}
\exp ( & \left.-2 \pi^{2} \sigma_{\mu_{0 x x}}^{2} u^{2}\right)-\left(\frac{\sigma_{\mu_{0 x y}}}{\sigma_{\mu_{0 x x}}}\right)^{2} \\
& \times \frac{I_{0 x y}}{\sqrt{I_{0 x x} I_{0 y y}}} \exp \left(-2 \pi^{2} \sigma_{\mu_{0 x y}}^{2} u^{2}\right) \geqslant 0,
\end{aligned}
$$

for any value of $\boldsymbol{u}$. On considering the cases $u=0$ and $u \rightarrow \infty$ we derive from equation (37) the following constraints:

$$
\sigma_{\mu_{0 x x}} \leqslant \sigma_{\mu_{0 x y}} \leqslant \sigma_{\mu_{0 x x}} \sqrt{\frac{\sqrt{I_{0 x x} I_{0 y y}}}{I_{0 x y}}}
$$

which represent the above-mentioned sufficient condition.

When $P(r, 0) \ll 1$, we see from equation (17) that $I_{0 x y} \ll \sqrt{I_{0 x x} I_{0 y y}}$. Then, according to equation (38), the values of $\sigma_{\mu_{0 x y}}$ can be much greater than $\sigma_{\mu_{0 x x}}$. 


\section{Propagation of PGSM beams}

We recall that, for the field generated by an ordinary GSM source (11), the following propagation formula holds (see e.g. [60]):

$$
\begin{array}{r}
J_{\mathrm{sc}}\left(\boldsymbol{r}_{1}, \boldsymbol{r}_{2}, z\right)=\frac{I_{0}}{F^{2}(z)} \exp \left[-\frac{\mathrm{i} k}{2 R(z)}\left(r_{1}^{2}-r_{2}^{2}\right)\right] \\
\quad \times \exp \left[-\frac{r_{1}^{2}+r_{2}^{2}}{4 \sigma_{I_{0}}^{2} F^{2}(z)}\right] \exp \left[-\frac{\left(\boldsymbol{r}_{1}-\boldsymbol{r}_{2}\right)^{2}}{2 \sigma_{\mu_{0}}^{2} F^{2}(z)}\right],
\end{array}
$$

where

$$
\begin{gathered}
F^{2}(z)=1+\frac{(\lambda z / \pi)^{2}}{4 \sigma_{I_{0}}^{2}}\left(\frac{1}{4 \sigma_{I_{0}}^{2}}+\frac{1}{\sigma_{\mu_{0}}^{2}}\right), \\
R(z)=z\left(1+\frac{1}{F^{2}(z)}\right) .
\end{gathered}
$$

There is a similar propagation formula [55] for each element of the BCP matrix describing the field generated by a PGSM source. More explicitly we have

$$
\begin{array}{r}
J_{\alpha \beta}\left(\boldsymbol{r}_{1}, \boldsymbol{r}_{2}, z\right)=\frac{I_{0 \alpha \beta}}{F_{\alpha \beta}^{2}(z)} \exp \left[-\frac{\mathrm{i} k}{2 R_{\alpha \beta}(z)}\left(r_{1}^{2}-r_{2}^{2}\right)\right] \\
\quad \times \exp \left[-\frac{r_{1}^{2}+r_{2}^{2}}{4 \sigma_{I_{0}}^{2} F_{\alpha \beta}^{2}(z)}\right] \exp \left[-\frac{\left(\boldsymbol{r}_{1}-\boldsymbol{r}_{2}\right)^{2}}{2 \sigma_{\mu_{0 \alpha \beta}}^{2} F_{\alpha \beta}^{2}(z)}\right],
\end{array}
$$

with

$$
\begin{gathered}
F_{\alpha \beta}^{2}(z)=1+\frac{(\lambda z / \pi)^{2}}{4 \sigma_{I_{0}}^{2}}\left(\frac{1}{4 \sigma_{I_{0}}^{2}}+\frac{1}{\sigma_{\mu_{0 \alpha \beta}}^{2}}\right), \\
R_{\alpha \beta}(z)=z\left(1+\frac{1}{F_{\alpha \beta}^{2}(z)}\right),
\end{gathered}
$$

and $\alpha, \beta=x, y$. Except for the case $\sigma_{\mu_{0 x x}}=\sigma_{\mu_{0 x y}}$, the expansion factors $F_{x x}(z)$ and $F_{x y}(z)$ are different at any $z>0$. Accordingly, the matrix elements $J_{x x}$ (or $J_{y y}$ ) and $J_{x y}$ change in a different way.

In order to see this, let us write the local polarization matrix (see section 2) $\hat{J}(\boldsymbol{r}, \boldsymbol{r}, z)$. Using equation (41) we find

$$
\begin{aligned}
& \hat{J}(\boldsymbol{r}, \boldsymbol{r}, z) \\
& =\left(\begin{array}{ll}
\frac{I_{0 x x}}{F_{x x}^{2}(z)} \exp \left[-\frac{r^{2}}{2 \sigma_{I_{0}}^{2} F_{x x}^{2}(z)}\right] & \frac{I_{0 x y}}{F_{x y}^{2}(z)} \exp \left[-\frac{r^{2}}{2 \sigma_{I_{0}}^{2} F_{x y}^{2}(z)}\right] \\
\frac{I_{0 x y}}{F_{x y}^{2}(z)} \exp \left[-\frac{r^{2}}{2 \sigma_{I_{0}}^{2} F_{x y}^{2}(z)}\right] & \frac{I_{0 y y}}{F_{x x}^{2}(z)} \exp \left[-\frac{r^{2}}{2 \sigma_{I_{0}}^{2} F_{x x}^{2}(z)}\right]
\end{array}\right) .
\end{aligned}
$$

On inserting from equation (43) into equation (6) we obtain the following law of variation for the degree of polarization upon propagation:

$$
\begin{aligned}
& P(\boldsymbol{r}, z)=\left\{\left(\frac{I_{0 x x}-I_{0 y y}}{I_{0 x x}+I_{0 y y}}\right)^{2}+\frac{4 I_{0 x y}^{2}}{\left(I_{0 x x}+I_{0 y y}\right)^{2}}\left[\frac{F_{x x}(z)}{F_{x y}(z)}\right]^{4}\right. \\
& \left.\quad \times \exp \left[-\frac{r^{2}}{\sigma_{I_{0}}^{2}}\left(\frac{1}{F_{x y}^{2}(z)}-\frac{1}{F_{x x}^{2}(z)}\right)\right]\right\}^{1 / 2} .
\end{aligned}
$$

Equation (44) shows that if $\sigma_{\mu_{0 x x}} \neq \sigma_{\mu_{0 x y}}$ and $I_{0 x y} \neq 0$ the degree of polarization, which is uniform at the plane $z=0$, will be non-uniform across a typical section $z=$ constant $>0$. It can be shown that the requirement that $P(r, z) \leqslant 1$ for any choice of $(\boldsymbol{r}, z)$ leads to further limitations for the values of $\sigma_{\mu_{0 x x}}$ and $\sigma_{\mu_{0 x y}}$ (see appendix B). In particular, it can be proved that, when the light field is completely polarized across the source plane $(P(\boldsymbol{r}, 0)=1)$, the radiated beam retains this property upon propagation, resulting in $\sigma_{\mu_{0 x x}}=\sigma_{\mu_{0 x y}}{ }^{5}$.

We have already pointed out the fact that the beam emitted by a PGSM source is absolutely undistinguishable from that generated by an ordinary, scalar GSM source if no anisotropic element is inserted in the beam path. In other terms, we can say that an interference scheme involving no anisotropic elements measures the trace of the BCP matrix, i.e. $J_{\text {eq }}\left(\boldsymbol{r}_{1}, \boldsymbol{r}_{2}, z\right.$ ) (see equation (7)). In order to demonstrate the effects of the offdiagonal elements of the BCP matrix, or even to measure them, one thus needs to use some anisotropic device, such as polarizers or waveplates.

The simplest scheme consists of a linear polarizer inserted across the beam path at a typical plane $z=$ constant. On denoting by $\vartheta$ the angle between the transmission axis of the polarizer and the $x$-axis, the transverse optical intensity after the polarizer $I_{\vartheta}(r, z)$ is given by equation (10), which, on considering for simplicity $I_{0 x x}=I_{0 y y}$, leads to

$$
I_{\vartheta}(\boldsymbol{r}, z)=J_{x x}(\boldsymbol{r}, \boldsymbol{r}, z)+J_{x y}(\boldsymbol{r}, \boldsymbol{r}, z) \sin 2 \vartheta .
$$

For example, on setting $\vartheta= \pm \pi / 4$, we obtain from equation (45)

$$
I_{ \pm \pi / 4}(\boldsymbol{r}, z)=J_{x x}(\boldsymbol{r}, \boldsymbol{r}, z) \pm J_{x y}(\boldsymbol{r}, \boldsymbol{r}, z),
$$

which, in the case of our PGSM beam, turns out to be

$$
\begin{gathered}
I_{ \pm \pi / 4}(\boldsymbol{r}, z)=I_{0 x x}\left[\frac{\exp \left[-\frac{r^{2}}{2 \sigma_{I_{0}}^{2} F_{x x}^{2}(z)}\right]}{F_{x x}^{2}(z)}\right. \\
\left. \pm \frac{\eta \exp \left[-\frac{r^{2}}{2 \sigma_{I_{0}}^{2} F_{x y}^{2}(z)}\right]}{F_{x y}^{2}(z)}\right],
\end{gathered}
$$

where $\eta=I_{0 x y} / I_{0 x x}$. Therefore, except for the case $\sigma_{\mu_{0 x y}}=$ $\sigma_{\mu_{0 x x}}$, the output intensity is no longer Gaussian, a feature one could hardly foresee looking at equation (12).

\section{Numerical example}

In this section it will be shown through a simple numerical example how the polarization features of PGSM beams change upon free propagation. On the other hand we will show how it is possible to distinguish PGSM beams from ordinary GSM beams when a linear polarizer is considered.

First of all it should be noticed that the local degree of polarization of a PGSM beam will change, showing different behaviours with $z$ and $r$, depending on the beam parameters. In order to illustrate the propagation characteristics of a PGSM beam and the differences between an ordinary GSM and PGSM beam we choose the simple case considered in the previous section. With this aim, we will assume that the total intensity at the source plane is normalized to unity and that $I_{0 x x}=I_{0 y y}$. The beam size at this plane is chosen as $\sigma_{I_{0}}=1 \mathrm{~mm}$ and the other beam parameters have been selected

5 It should be noted that the condition $P(r, z) \leqslant 1$ can be traced back to the Schwartz inequality (4) [1]. 


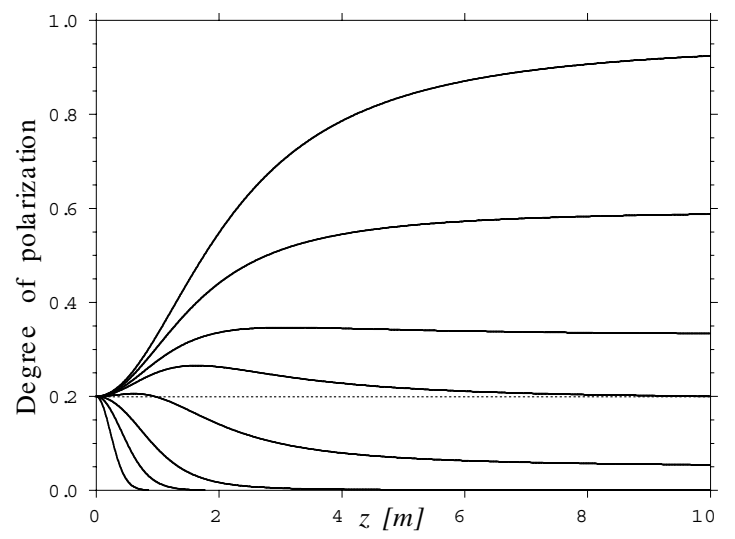

Figure 1. Polarization degree of a PGSM beam as a function of the propagation distance $z$ for several values of $r / \sigma_{I}(z)$ (from the top to the bottom $\left.r / \sigma_{I}(z)=0,0.5,0.75,0.92,1.25,2,3,5\right)$. Beam parameters are $I_{0 x x}=I_{0 y y}=1 / 2, I_{0 x y}=0.1, \sigma_{I o}=1 \mathrm{~mm}$, $\sigma_{\mu_{0 x x}}=\sigma_{\mu_{0 y y}}=0.1 \mathrm{~mm}, \sigma_{\mu_{0 x y}}=0.2 \mathrm{~mm}$ and $\lambda=632.8 \mathrm{~nm}$.

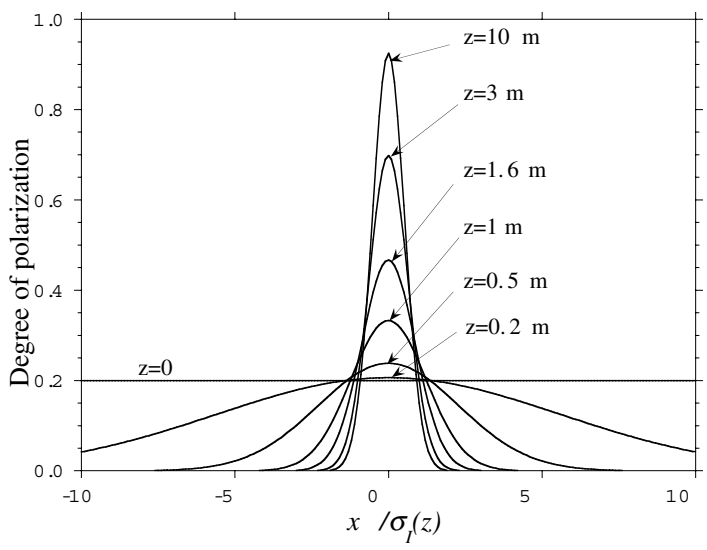

Figure 2. Polarization degree of a PGSM beam as a function of the scaled variable $x / \sigma_{I}(z)$ at different propagation distances $z$. Due to the rotational symmetry of the beam only the $x$-section $(y=0)$ is shown. Beam parameters are as in figure 1.

in such a way as to satisfy equations (13), (14), (16), (18), (29) and (38). In particular we set $I_{0 x x}=I_{0 y y}=1 / 2, I_{0 x y}=0.1$, $\sigma_{\mu_{0 x x}}=\sigma_{\mu_{0 y y}}=0.1 \mathrm{~mm}$ and $\sigma_{\mu_{0 x y}}=0.2 \mathrm{~mm}$. In such a case the beam is partially polarized. If we use the decomposition of a given wave into a unpolarized and a polarized portion which are mutually independent [2], then its totally polarized component is linear with azimuth $+\pi / 4$.

Figure 1 shows the local degree of polarization $P(\rho, z)$, calculated from equation (44), versus the propagation distance $z$ for various values of $\rho$. We used the scaled variable $\rho=r / \sigma_{I}(z)$, where $r$ is the radial coordinate and $\sigma_{I}(z)$ is the beam width at each plane $z=$ constant. As can be seen, the polarization at the source plane is uniform for all the points at the transversal section of the beam with the same value $P(\rho, 0)=0.2$. As the beam propagates in free space the degree of polarization becomes different from point to point of the cross section of the beam. The degree of polarization never remains constant along $z$, although in some cases it is possible to obtain the same value of $P(\rho, z)$ at both the far field and the source plane (see figure 1 for $\rho=0.92$ ).

In order to compare the degree of polarization distribution across the transversal section of the beam for different values
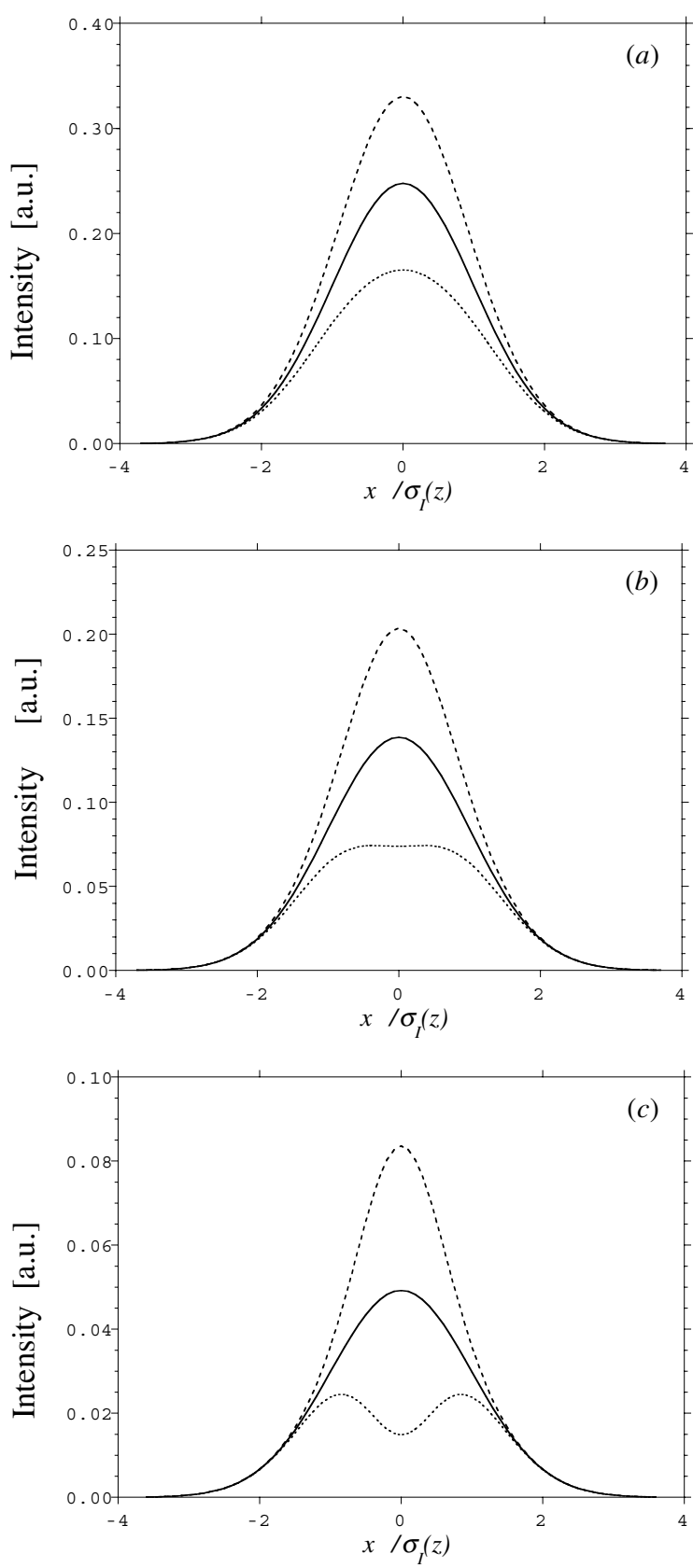

Figure 3. PGSM beam intensity after a linear polarizer versus $x / \sigma_{I}(z)$ at different angles with respect to the $x$-axis: $\theta=0$ (solid curves), $\theta=\pi / 4$ (dashed curves) and $\theta=-\pi / 4$ (dotted curves), placing the polarizer at $(a) z=1 \mathrm{~m},(b) z=1.6 \mathrm{~m}$ and $(c) z=3 \mathrm{~m}$. Beam parameters are as in figures 1 and 2 .

of $z$ we have plotted $P(\rho, z)$ along the $x$-axis $(y=0)$ (see figure 2). It is clear from this figure that the non-uniformity of the degree of polarization at the beam transversal section is different for each plane $z=$ constant. It starts from a constant distribution at $z=0$, changing to a Gaussian distribution, whose variance decreases as $z$ is increased.

Finally we show the effect of a linear polarizer on a PGSM beam. As pointed out above, it is not possible to distinguish a PGSM from an ordinary GSM beam when only beam intensity measures are performed, but it is necessary to introduce some anisotropic element such as a polarizer. The intensity of a 
PGSM beam at the output of a polarizer is calculated from equation (45) for our beam and for three different values of $\theta$. The polarizer is placed at three distances $z$ from the source plane. The results are plotted in figure 3. For $\theta=0$ the beam intensity profile is Gaussian at each plane $z$ whereas for $\theta= \pm \pi / 4$ we have a sharper or flatter beam profile.

Note that as the beam propagates its central region presents a higher degree of polarization (see figure 2). This means that the fraction of the totally polarized component of the beam is increased and remains linearly polarized at $+\pi / 4$. This fact accounts for the shape of the curves of figure 3 , where an intensity reduction (hole) is seen at the centre of the beam when the polarizer is placed at $\theta=-\pi / 4$ whereas a higher value of the intensity at the centre is observed for $\theta=+\pi / 4$.

\section{Conclusions}

We have considered a six-parameter class of partially polarized Gaussian Schell-model (PGSM) sources with uniform polarization as well as the beams generated by such sources. We found that the choice of the six parameters is restricted by certain constraints. Upon propagation the polarization across a typical beam section generally becomes non-uniform. At an intuitive level, this can presumably be accepted as due to a different correlation length of the diagonal elements as compared with the off-diagonal ones. More surprising is the fact that on rotating a linear polarizer inserted in the beam path we see the central region of the intensity pattern turn into a dip or a hill with respect to the Gaussian shape. In a sense, the beam behaves as the incoherent superposition of two non-Gaussian shaped beams with orthogonal polarization. When two such beams are superposed and no anisotropic optical element is used the result is just an ordinary GSM beam. Neither the intensity pattern nor the equivalent mutual intensity distribution would allow us to realize that the beam has a richer structure than the ordinary GSM beam. The physical difference between the two beams is accounted for by the simple BCP matrix treatment.

As we said previously, the unrestricted family of PGSM beams is specified by nine parameters instead of six. Indeed, this set is expected to exhibit an even richer behaviour than the set considered in this paper. Criteria for the correct choice of the nine parameters remain to be found and a full scope description of the corresponding propagation features has to be developed.

\section{Acknowledgments}

GP acknowledges support from the PB97-0295 project and from the programme 'Becas Internacionales Universidad Complutense/Flores Valles'.

\section{Appendix A. Removing the phase of $I_{0 x y}$}

Let us consider the partially polarized GSM source passing through a waveplate whose Jones matrix, say $\hat{T}_{\mathrm{WP}}$, is of the form

$$
\hat{T}_{\mathrm{WP}}=\left(\begin{array}{cc}
1 & 0 \\
0 & \exp (\mathrm{i} \Delta \varphi)
\end{array}\right),
$$

where $\Delta \varphi$ denotes the phase delay introduced by the waveplate. The local BCP matrix across the source plane of our partially polarized GSM source turns out to be

$$
\begin{aligned}
& \hat{J}\left(\boldsymbol{r}_{1}, \boldsymbol{r}_{2} ; 0\right)=\exp \left(-\frac{r_{1}^{2}+r_{2}^{2}}{4 \sigma_{I o}^{2}}\right) \\
& \times\left(\begin{array}{cc}
I_{0 x x} \exp \left[-\frac{\left(r_{1}-r_{2}\right)^{2}}{2 \sigma_{\mu_{0 x x}}^{2}}\right. & M_{0 x y} \exp \left(\mathrm{i}_{0 x y}\right) \exp \left[-\frac{\left(r_{1}-r_{2}\right)^{2}}{2 \sigma_{\mu_{0 x y}}^{2}}\right] \\
M_{0 x y} \exp \left(-\mathrm{i} \Phi_{0 x y}\right) \exp \left[-\frac{\left(r_{1}-r_{2}\right)^{2}}{2 \sigma_{\mu_{0 x y}}^{2}}\right] & I_{0 y y} \exp \left[-\frac{\left(r_{1}-r_{2}\right)^{2}}{2 \sigma_{\mu_{0 x x}}^{2}}\right]
\end{array}\right),
\end{aligned}
$$

where we set $I_{0 x y}=M_{0 x y} \exp \left(\mathrm{i} \Phi_{0 x y}\right)$.

On substituting equations (A.1) and (A.2) into (9), the BCP matrix after the passage through the waveplate turns out to be

$$
\begin{aligned}
& \hat{J}^{\prime}\left(\boldsymbol{r}_{1}, \boldsymbol{r}_{2} ; 0\right)=\hat{T}_{\mathrm{WP}}^{\dagger} \hat{J}\left(\boldsymbol{r}_{1}, \boldsymbol{r}_{2}, z\right) \hat{T}_{\mathrm{WP}} \\
& =\exp \left(-\frac{r_{1}^{2}+r_{2}^{2}}{4 \sigma_{I o}^{2}}\right) \\
& \times\left(\begin{array}{cc}
I_{0 x x} \exp \left[-\frac{\left(r_{1}-r_{2}\right)^{2}}{2 \sigma_{\mu_{0 x x}}^{2}}\right. & M_{0 x y} \exp \left(\mathrm{i}_{0 x y}+\mathrm{i} \Delta \varphi\right) \exp \left[-\frac{\left(r_{1}-r_{2}\right)^{2}}{2 \sigma_{\mu_{0 x y}}^{2}}\right] \\
M_{0 x y} \exp \left(-\mathrm{i} \Phi_{0 x y}-\mathrm{i} \Delta \varphi\right) \exp \left[-\frac{\left(r_{1}-r_{2}\right)^{2}}{2 \sigma_{\mu_{0 x y}}^{2}}\right] & I_{0 y y} \exp \left[-\frac{\left(r_{1}-r_{2}\right)^{2}}{2 \sigma_{\mu_{0 x x}}^{2}}\right]
\end{array}\right),
\end{aligned}
$$

from which, on setting $\Delta \varphi=-\Phi_{0 x y}$, the phase of $I_{0 x y}$ can be removed.

\section{Appendix B. Further constraint of BCP parameters}

We derive a further necessary condition for the values of $\sigma_{\mu_{0 x x}}$ and $\sigma_{\mu_{0 x y}}$, following from the fact that the degree of polarization $P(r, z)$ has to be less than or equal to unity everywhere in space [1]. First of all, we see from equation (44) that, for a fixed value of $z$, the maximum value of the degree of polarization is reached at $r=0$. This maximum varies with $z$ according to the following law:

$$
P(0, z)=\sqrt{\left(\frac{I_{0 x x}-I_{0 y y}}{I_{0 x x}+I_{0 y y}}\right)^{2}+\frac{4 I_{0 x y}^{2}}{\left(I_{0 x x}+I_{0 y y}\right)^{2}}\left[\frac{F_{x x}(z)}{F_{x y}(z)}\right]^{4}} .
$$

In the limit of very large values of $z$, equation (B.1) gives

$$
\begin{aligned}
\lim _{z \rightarrow \infty} P(0, z) & \sqrt{\left(\frac{I_{0 x x}-I_{0 y y}}{I_{0 x x}+I_{0 y y}}\right)^{2}+\frac{4 I_{0 x y}^{2}}{\left(I_{0 x x}+I_{0 y y}\right)^{2}}\left(\frac{\frac{1}{4 \sigma_{I_{0}}^{2}}+\frac{1}{\sigma_{\mu_{0 x x}}^{2}}}{\frac{1}{4 \sigma_{I_{0}}^{2}}+\frac{1}{\sigma_{\mu_{0 x y}}^{2}}}\right)^{2}},
\end{aligned}
$$

where use has been made of equation (42). In particular, it is possible to show that limit (B.2) constitutes the maximum for the degree of polarization, i.e.

$$
P(\boldsymbol{r}, z) \leqslant P(0, \infty),
$$

for any choice of $(\boldsymbol{r}, z)$. Thus, on imposing that $P(0, \infty) \leqslant 1$, after simple algebra we obtain the following inequality:

$$
\sigma_{\mu_{0 x y}} \leqslant \sigma_{\mu_{0 x x}} \gamma \sqrt{\frac{4 \sigma_{I o}^{2}+\sigma_{\mu_{0 x y}}^{2}}{4 \sigma_{I o}^{2}+\sigma_{\mu_{0 x x}}^{2}}}
$$


where $\gamma=\sqrt{I_{0 x x} I_{0 y y}} / I_{0 x y}$. Equation (B.4) represents a necessary condition. By solving it with respect to $\sigma_{\mu_{0 x y}}$, it can be shown that equation (B.4) becomes

$$
\sigma_{\mu_{0 x y}} \leqslant \frac{2 \gamma \sigma_{I_{0}} \sigma_{\mu_{0 x x}}}{\sqrt{4 \sigma_{I_{0}}^{2}-\left(\gamma^{2}-1\right) \sigma_{\mu_{0 x x}}^{2}}},
$$

for those values of $\sigma_{I_{0}}, \gamma, \sigma_{\mu_{0 x x}}$, for which the argument of the square root in equation (B.5) is greater than zero. For other values of $\sigma_{I_{0}}, \gamma, \sigma_{\mu_{0 x x}}$, it is possible to see that condition (B.4) is always satisfied. An important consequence of equation (B.5) is that, when the degree of polarization across the source plane is equal to unity, i.e. when $\gamma=1$ (see equation (17)), $\sigma_{\mu_{0 x y}}$ cannot exceed $\sigma_{\mu_{0 x x}}$. According to equation (28), this means that the only possible choice is $\sigma_{\mu_{0 x y}}=\sigma_{\mu_{0 x x}}$, so the radiated beam remains completely polarized at any transverse plane.

\section{References}

[1] Mandel L and Wolf E 1995 Optical Coherence and Quantum Optics (Cambridge: Cambridge University Press)

[2] Born M and Wolf E 1999 Principles of Optics 7th edn (Cambridge: Cambridge University Press)

[3] Friberg A T (ed) 1993 Selected Papers on Coherence and Radiometry (SPIE Milestone Series 69) (Bellingham, WA: SPIE)

[4] Collett E and Wolf E 1978 Is complete coherence necessary for the generation of highly directional light beams? Opt. Lett. 2 27-9

[5] Gori F and Palma C 1978 Partially coherent sources which give rise to highly directional light beams Opt. Commun. 27 $185-8$

[6] Foley J T and Zubairy M S 1978 The directionality of Gaussian Schell-model beams Opt. Commun. 26 297-300

[7] Saleh B E A 1979 Intensity distribution due to a partially coherent field and the Collett-Wolf equivalence theorem in the Fresnel zone Opt. Commun. 30 135-8

[8] Friberg A T and Sudol R J 1982 Propagation parameters of Gaussian Schell-model beams Opt. Commun. 41 383-7

[9] Martínez-Herrero R and Mejías P M 1982 Relation among planar sources that generate the same radiant intensity at the output of a general optical system J. Opt. Soc. Am. 72 765-9

[10] Gamliel 1986 Radiation efficiency of planar Gaussian Schell-model sources Opt. Commun. 60333

[11] Zahid M and Zubairy M S 1987 Second-order coherence properties of beams generated by a generalized Schell-model source Opt. Commun. 64496

[12] He, Turunen J and Friberg A T 1988 Propagation and imaging experiments with Gaussian Schell-model beams $O p t$. Commun. 67245

[13] Simon R and Tamir T 1989 Nonspecular phenomena in partly coherent beams reflected by multilayered structures $J$. Opt. Soc. Am. A 618

[14] Nugent K A 1990 A generalization of Schell's theorem Opt. Commun. 79267

[15] Serna J, Martínez-Herrero R and Mejías P M 1992 Beam quality dependence on the coherence length of Gaussian Schell-model fields propagating through ABCD optical systems J. Mod. Opt. 39625

[16] Cincotti G, De Santis P, Guattari G and Palma C 1994 Propagation of partially coherent beams in a periodic sequence of lenses and Gaussian apertures J. Eur. Opt. Soc. A 3561

[17] Yoshimura H, Takas N and Asakura T 1994 Equiambiguity-function ellipse of Gaussian Schell-model beams J. Opt. Soc. Am. A 111136

[18] Castañeda R and Medina F F 1997 Schell-model beams and interference fields Optik 105 88-92
[19] Gori F 1980 Collett-Wolf sources and multimode laser Opt. Commun. 34 301-5

[20] Starikov A and Wolf E 1982 Coherent-mode representation of Gaussian Schell-model sources and of their radiation fields J. Opt. Soc. Am. 72 923-8

[21] Gase R 1991 The multimode laser radiation as a Gaussian Schell-model beam J. Mod. Opt. 38 1107-15

[22] Lüet B, Zhang B, Cai B and Yang C 1993 A simple method for estimating the number of effectively oscillating modes and weighting factors of mixed-mode laser beams behaving like Gaussian Schell-model beams Opt. Commun. 10149

[23] Wolf E 1982 New theory of partial coherence in the space-frequency domain. Part I: Spectra and cross-spectra of steady-state sources J. Opt. Soc. Am. 72 343-51

[24] Simon R and Mukunda N 1993 Twisted Gaussian Schell-model beams J. Opt. Soc. Am. A 1095

[25] Simon R, Sundar K and Mukunda N 1993 Twisted Gaussian Schell-model beams. I. Symmetry structure and normal-mode spectrum J. Opt. Soc. Am. A 102008

[26] Sundar K, Simon R and Mukunda N 1993 Twisted Gaussian Schell-model beams. II. Spectrum analysis and propagation characteristics J. Opt. Soc. Am. A 102017

[27] Ambrosini D, Bagini V, Gori F and Santarsiero M 1994 Twisted Gaussian Schell-model beams: a superposition model J. Mod. Opt. 41 1391-9

[28] Friberg A T, Tervonen E and Turunen J 1994 Focusing of twisted Gaussian Schell-model beams Opt. Commun. 106 $127-32$

[29] Friberg A T, Tervonen E and Turunen J 1994 Interpretation and experimental demonstration of twisted Gaussian Schell-model beams J. Opt. Soc. Am. A 111818

[30] Gori F, Santarsiero M, Borghi R and Vicalvi S 1998 Partially coherent sources with helicoidal modes J. Mod. Opt. 45 $539-54$

[31] Mascello A, Perrone M R and Palma C 1997 Coherence evolution of laser beams in cavities with variable-reflectivity mirrors J. Opt. Soc. Am. A 141890

[32] Garnier J, Gouédard C and Videau L 2000 Propagation of a partially coherent beam under the interaction of small and large scales Opt. Commun. 176 281-98

[33] Tidwell S C, Ford D H and Kimura W D 1990 Generating radially polarized beams interferometrically Appl. Opt. 29 2334-9

[34] Erdogan T, King O, Wicks G W, Hall D G, Anderson E and Rooks M J 1992 Circularly symmetric operation of a concentric-circle-grating surface emitting $\mathrm{AlGaAs} / \mathrm{GaAs}$ quantum well semiconductor laser Appl. Phys. Lett. 60 1921-3

[35] Higgins T V 1992 Spiral waveplate design produces radially polarized laser light Laser Focus World 28 18-20

[36] Tidwell S C, Kim G H and Kimura W D 1993 Efficient radially polarized laser beam generation with double interferometer Appl. Opt. 32 5223-8

[37] James D F V 1994 Change of polarization of light beam on propagation in free-space J. Opt. Soc. Am. A 111641

[38] Jordan R H and Hall D G 1994 Free-space azimuthal paraxial wave equation: the azimuthal Bessel-Gauss solution Opt. Lett. 19 427-9

[39] Hall D G 1996 Vector-beam solution of Maxwell's wave equation Opt. Lett. 21 9-12

[40] Greene P L and Hall D G 1998 Properties and diffraction of vector Bessel-Gauss beams J. Opt. Soc. Am. A 15 3020-7

[41] Olson C, Greene P L, Wicks G W, Hall D G and Rishton S 1998 High-order azimuthal spatial modes of concentric-circle-grating surface-emitting semiconductor lasers Appl. Phys. Lett. 72 1284-6

[42] Martínez-Herrero R, Mejías P M and Movilla J M 1997 Spatial characterization of partially polarized beams Opt. Lett. 22 206-8

[43] Movilla J M, Piquero G, Martínez-Herrero R and Mejías P M 1998 Parametric characterization of non-uniformly polarized beams Opt. Commun. 149 230-4 
[44] Piquero G, Movilla J M, Mejías P M and Martínez-Herrero R 1999 Degree of polarization of non-uniformly partially polarized beams: a proposal Opt. Quantum Electron. 31 223-5

[45] Piquero G, Movilla J M, Martínez-Herrero R and Mejías P M 1999 Beam quality of partially polarized beams propagating through lens-like birefringent elements J. Opt. Soc. Am. A $162666-8$

[46] Tovar A A 1998 Production and propagation of cylindrically polarized Laguerre Gaussian laser beams J. Opt. Soc. Am. A 152705

[47] Nikolova L, Ivanov M, Todorov T and Stoyanov S 1993 Spectrophotopolarimeter: a simplified version for real-time measurement at selected wavelengths Bulgarian J. Phys. 20 46-54

[48] Rochon P, Drnoyan V and Natansohn A 2000 Polarization holographic gratings in azopolymers for detecting and producing circularly polarized light Proc. 1998 Int. Conf. on Applications of Photonic Technology III: Closing the Gap between Theory, Developments, and Applications (SPIE 3491) ed G A Lampropoulos and R A Lessard (Bellingham, WA: SPIE)

[49] Gori F 1999 Measuring Stokes parameters by means of a polarization grating Opt. Lett. 24584

[50] Someda C G 2000 Far field of polarization gratings Opt. Lett. 251657
[51] Kawano K, Ishii T, Minabe J, Niitsu T, Nishikata Y and Baba K 1999 Holographic recording and retrieval of polarized light by use of polyester containing cyanoazobenzene units in the side chain Opt. Lett. 24 1269-71

[52] Ferrari J A, Frins E M and Dultz W 1998 Complex self-coherence function determination using geometric phase techniques Opt. Commun. 152252

[53] Seshadri S R 1999 Partially coherent Gaussian Schell-model electromagnetic beam J. Opt. Soc. Am. A 16 1373-80

[54] Gori F 1998 Matrix treatment for partially polarized, partially coherent beams Opt. Lett. $\mathbf{2 3} 241$

[55] Gori F, Santarsiero M, Vicalvi S, Borghi R and Guattari G 1998 Beam coherence-polarization matrix J. Eur. Opt. Soc. A 7 941-51

[56] Gori F, Santarsiero M, Borghi R and Guattari G 1999 The irradiance of partially polarized beams in a scalar treatment Opt. Commun. 163 159-63

[57] Wolf E 1959 Coherence properties of partially polarized electromagnetic radiation Nuovo Cimento 13 1165-81

[58] Azzam R M A and Bashara N M 1986 Ellipsometry and Polarized Light (Amsterdam: North-Holland)

[59] Brosseau C 1998 Fundamentals of Polarized Light (New York: Wiley)

[60] Gori F 1983 Mode propagation of the field generated by Collett-Wolf sources Opt. Commun. 46 149-54 